\title{
Numerical Simulation Research on the Interactive Propagation of Hydraulic Fractures and Natural Fractures
}

\author{
Asiri Waleed Abdullah I ${ }^{1 *}$, Ma Xiaoming ${ }^{1}$ \\ ${ }^{1}$ School of Mechanical and Automotive Engineering, South China University of Technology
}

\begin{abstract}
In order to study the fracture propagation behavior after the interaction between hydraulic fractures and natural fractures, the cohensive unit of Abaqus finite element software is used to simulate the influence of natural fractures in the rock on the propagation behavior of hydraulic fractures. The simulation results show that when the approach angle is kept constant, as the in-situ stress difference increases, hydraulic fractures are more likely to penetrate the natural fractures and expand along the direction of the maximum horizontal principal stress; while the stress difference remains the same, as the approach angle increases, hydraulic fractures are easier to penetrate natural fractures and expand along the direction of maximum horizontal principal stress. When the approach angle is kept constant, as the tensile strength of natural fractures increases, hydraulic fractures are more likely to penetrate the natural cracks and expand along the direction of the maximum horizontal principal stress; while the tensile strength of natural cracks remains unchanged, as the approach angle increases, the probability of forming a complex fracture network between hydraulic fractures and natural fractures is reduced, and it is easier to penetrate the natural fractures and expand along the direction of the maximum horizontal principal stress.
\end{abstract}

\section{Introduction}

With the continuous advancement of fracturing technology, the fracturing design concept has gradually developed to the stage of volume simulation. The concept is to use segmented multi-cluster perforation, high displacement, large liquid volume, low viscosity liquid and steering materials to make hydraulic fractures communicate with more natural fractures to form a fracture network, which significantly increases the contact area between fractures and reservoirs, maximizes the volume of reconstruction, and greatly increases production $^{[1]}$. The existence of natural fractures makes the propagation behavior of hydraulic fractures mainly include phenomena such as being trapped by natural fractures or penetrating natural fractures ${ }^{[2]}$. How the hydraulic fractures intersect with natural fractures is of great significance to predict the formation process of complex fracture networks. Some scholars have studied various factors influencing the morphology of hydraulic fractures through numerical simulations ${ }^{[3-4]}$, but the influence of natural fractures on the propagation of hydraulic fractures needs to be studied in depth, and laboratory experimental studies are difficult to study the dynamic stress distribution of hydraulic fractures. The repeatability of the experiment is not strong ${ }^{[5]}$. This paper uses ABAQUS finite element software to simulate the propagation behavior of hydraulic fractures after encountering natural fractures, and analyzes the influence of natural fractures' tensile strength and fracture inclination on the extension of hydraulic fractures.

\section{Numerical simulation research on the interactive propagation of hydraulic fractures and natural fractures}

When hydraulic fractures encounter natural fractures in the process of propagation, if there is a certain angle between them, the maximum tensile stress criterion is used to judge whether the natural fractures can open, that is, when the maximum tensile stress of the fracturing fluid acting on the natural fracture surface exceeds the tensile strength of the natural fractures ,they are opened and hydraulic fractures will turn to propagate along the natural fractures. If the stress generated by the fracturing fluid in the fractures is not enough to open the natural fractures, the hydraulic fractures will directly penetrate the natural fractures and expand forward.

Using ABAQUS numerical simulation software's cohesive unit to simulate the influence of natural fractures in rock on the propagation of hydraulic fractures. A two-dimensional numerical analysis model of $20 \mathrm{~m} \times 20 \mathrm{~m}$ is established, and natural fractures and hydraulic fractures are prefabricated by cohesive units. The length of the natural fractures is $6 \mathrm{~m}$, and the angles of the natural fractures are $45^{\circ}, 60^{\circ}, 75^{\circ}$, and $90^{\circ}$. The specific parameters are as follows .

\footnotetext{
* Corresponding author: 2363760116@qq.com
} 
Table 1. Model parameters.

\begin{tabular}{|c|c|}
\hline parameter & value \\
\hline Approach angle/ & 45, 60,75 \\
\hline Stress difference/MPa & $0,2 、 4,6$ \\
\hline rate $/ \mathrm{m} 2 \cdot \mathrm{s}^{-1}$ & 0.001 \\
\hline Fracturing fluid viscosity $/ \mathrm{Pa} \cdot \mathrm{s}$ & 0.001 \\
\hline Rock elastic modulus/MPa & 15 \\
\hline Poisson's ratio & 0.25 \\
\hline Rock tensile strength/MPa & 6 \\
\hline $\begin{array}{c}\text { Tensile strength of natural } \\
\text { cracks/MPa }\end{array}$ & $1,2 、 3,4$ \\
\hline $\begin{array}{c}\text { Permeability coefficient } / \mathrm{m} \cdot \mathrm{s}^{-1} \\
\text { Filtration }\end{array}$ & $1 \times 10^{-7}$ \\
\hline coefficient $/ \mathrm{m} \cdot(\mathrm{Pa} \cdot \mathrm{s})$ & $1 \times 10^{-14}$ \\
\hline $\begin{array}{c}\text { Initial void ratio } \\
\text { Model size } / \mathrm{m}\end{array}$ & 0.1 \\
\hline
\end{tabular}

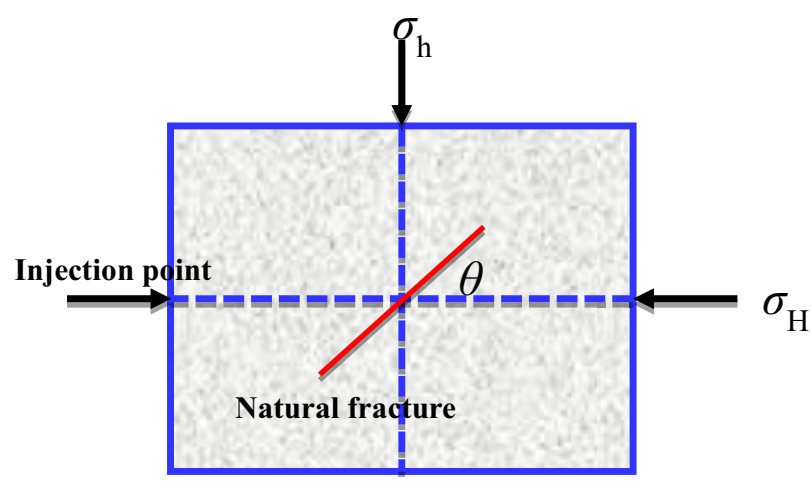

Fig. 1. Schematic diagram of the model.

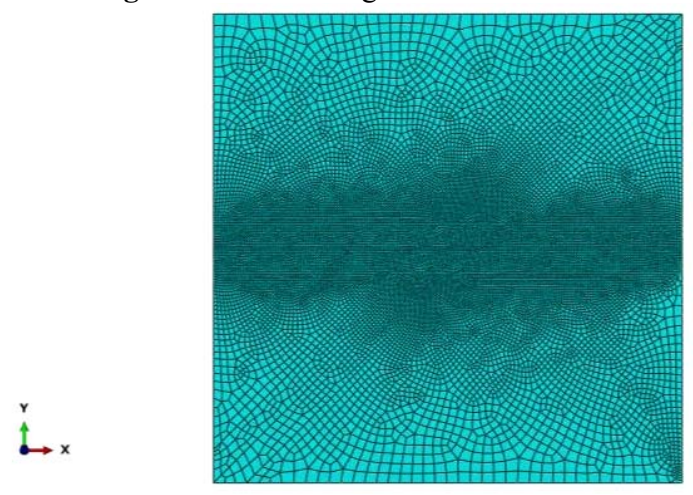

Fig. 2. Two-dimensional numerical analysis model.

\section{Analysis of numerical simulation results}

\subsection{The influence of in-situ stress difference on hydraulic fracture propagation}

By setting different combinations of in-situ stress difference and approach angle, the fracture propagation behavior of hydraulic fractures encountering natural fractures in different combinations is studied. When the approach angle is $60^{\circ}$ and the stress difference is $0 \mathrm{MPa}$, $2 \mathrm{MPa}, 4 \mathrm{MPa}$, and $6 \mathrm{MPa}$, the results of fracture propagation are shown in Fig 3,4,5,6.
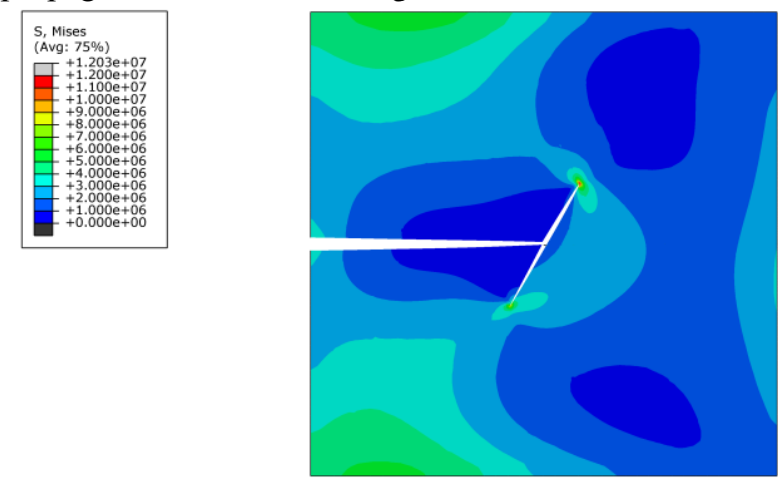

Fig. 3. the fracture propagation situation when the approach angle is $60^{\circ}$ and the stress difference is $0 \mathrm{MPa}$.

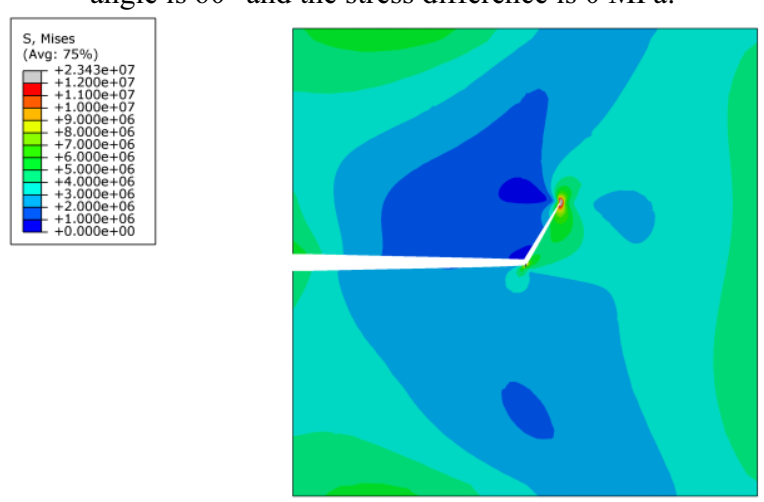

Fig. 4. the fracture propagation situation when the approach angle is $60^{\circ}$ and the stress difference is $2 \mathrm{MPa}$.
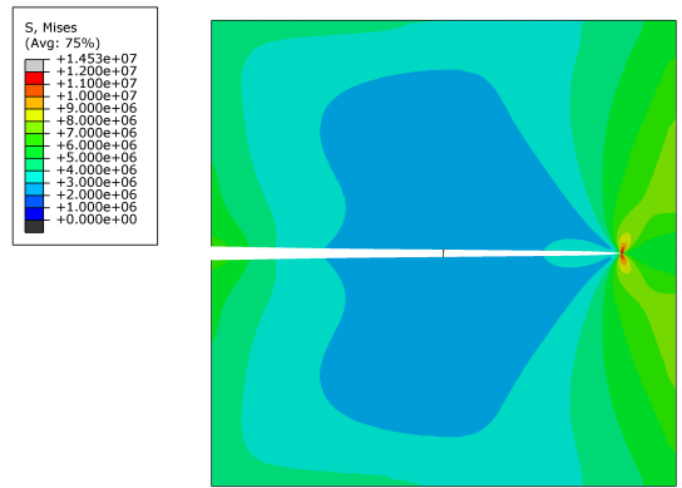

Fig. 5. the fracture propagation situation when the approach angle is $60^{\circ}$ and the stress difference is $4 \mathrm{MPa}$. 


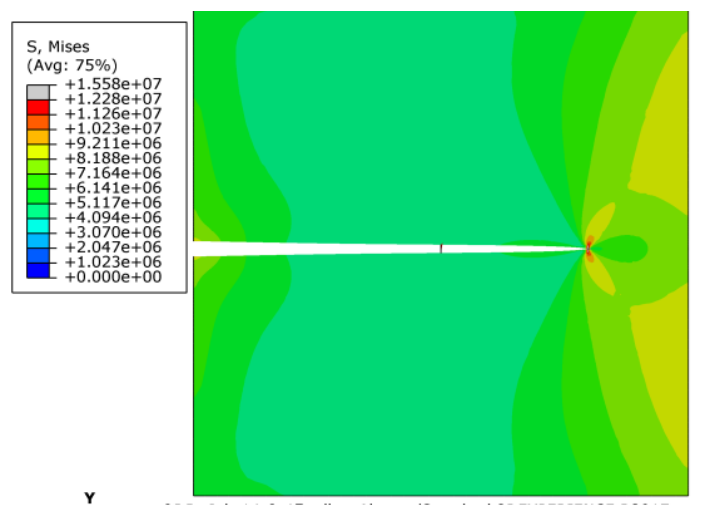

Fig. 6. the fracture propagation situation when the approach angle is $60^{\circ}$ and the stress difference is $6 \mathrm{MPa}$.

Table 2. Propagation behavior of hydraulic fractures under different stress differences and different approach angles.

\begin{tabular}{|c|c|c|c|c|}
\hline $\begin{array}{c}\text { approach } \\
\text { angle } \\
\text { stress }\end{array}$ & 45 & 60 & 75 & 90 \\
\hline 0 & capture & capture & capture & traverse \\
\hline 2 & capture & capture & traverse & traverse \\
\hline 4 & capture & traverse & traverse & traverse \\
\hline 6 & traverse & traverse & traverse & traverse \\
\hline
\end{tabular}

As can be seen from the above figure, when the approach angle is $60^{\circ}$, the hydraulic fractures will expand along the direction of the maximum horizontal principal stress when they do not encounter natural fractures. When the in-situ stress difference is $0 \mathrm{MPa}$, the hydraulic fractures will be captured by the natural fractures after encountering natural fractures, and continue to expand along the two wings of the natural fracture; when the in-situ stress difference is $2 \mathrm{MPa}$, the hydraulic fracture encounters the natural fracture and then is captured by the natural fracture, and continues to expand along the upper wing of the natural fracture; when the in-situ stress difference is $4 \mathrm{MPa}$, the hydraulic fracture crosses the natural fracture and continues to expand. The numerical simulation results in Table 2 show that when the approach angle is kept constant, as the ground stress difference increases, the ability of hydraulic fractures to communicate with natural fractures becomes worse and worse, and it is easier to penetrate natural fractures and expand along the direction of the maximum horizontal principal stress. The probability of forming a complex fracture network is reduced; when the stress difference remains the same, the increase of the approach angle decreases the ability of hydraulic fractures communication with natural fractures, and hydraulic fractures are easier to penetrate natural fractures and then expand along the maximum horizontal principal stress direction. Mainly as the approach angle increases, the opening pressure of the fracturing fluid on the natural fractures decreases, making it difficult for natural fractures to open.

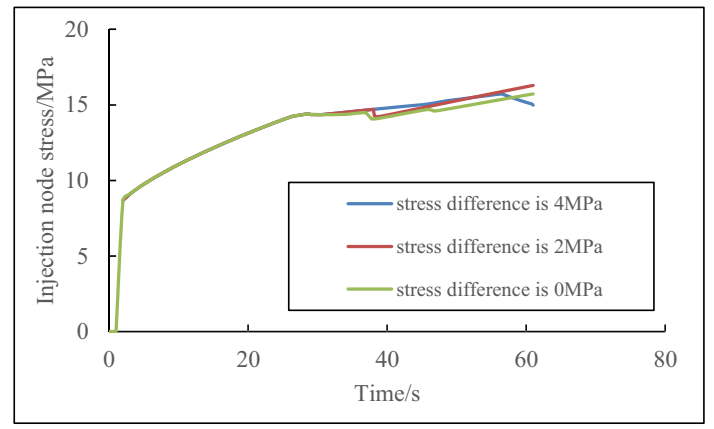

Fig. 7. the injection node stress situation when the approach angle is $60^{\circ}$.

It can be seen from Fig.7 that the injection point stress gradually increases with the continuous injection of fracturing fluid before intersecting with natural fractures. the growth trend of the injection point stress under different stress differences is the same. After the hydraulic fracture intersects the natural fracture, when the stress difference is $0 \mathrm{MPa}$ and $2 \mathrm{MPa}$, The curves suddenly drop for a while, and then continue to increase. This is because the hydraulic fracture intersects with the natural fracture and opens the natural fracture, which makes the fracturing fluid in the hydraulic fracture suddenly flow into the natural fracture, and the volume of the fracturing fluid in the hydraulic fracture decreases instantaneously, which leads to the decrease of the injection point stress suddenly. As the fracturing fluid continues to be injected, the fracture width gradually increases, and the increasing trend of $0 \mathrm{MPa}$ is more gentle than the curve of $2 \mathrm{MPa}$, because it is easier to extend along the natural fracture in the reverse directionwhen the stress difference is $0 \mathrm{MPa}$, while the stress difference is $2 \mathrm{MPa}$, the hydraulic fracture can't extend along the natural fracture in the opposite direction. When the horizontal stress difference is $4 \mathrm{MPa}$, the hydraulic fracture directly penetrates the natural fracture, so the injection point stress hasn't changed much.

\subsection{The influence of the tensile strength of natural fractures on the propagation of hydraulic fractures}

By setting different combinations of natural fracture tensile strength and approach angle, the propagation behavior of hydraulic fractures in different combinations after encountering natural fractures is studied. The tensile strength of natural fracture is $2 \mathrm{MPa}$, and the fracture propagation results are shown in Figure $8,9,10,11$ when the approach angles are $45^{\circ}, 60^{\circ}$ and $75^{\circ}$. 


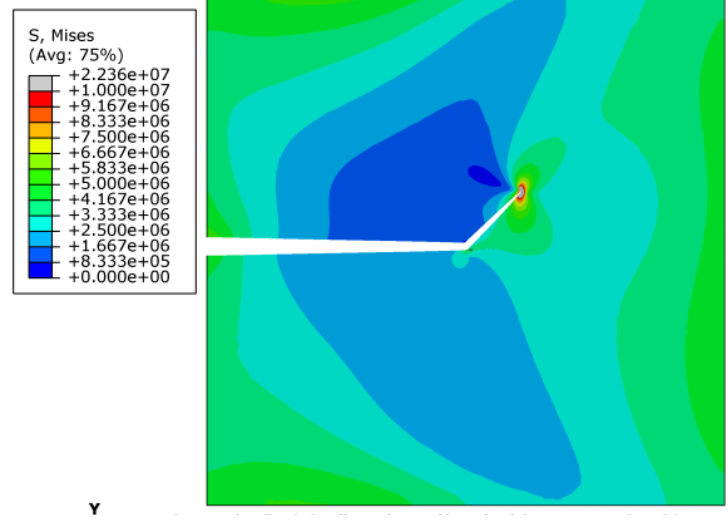

Fig. 8. the fracture propagation situation when the approach angle is $45^{\circ}$ and the tensile strength is $2 \mathrm{MPa}$.

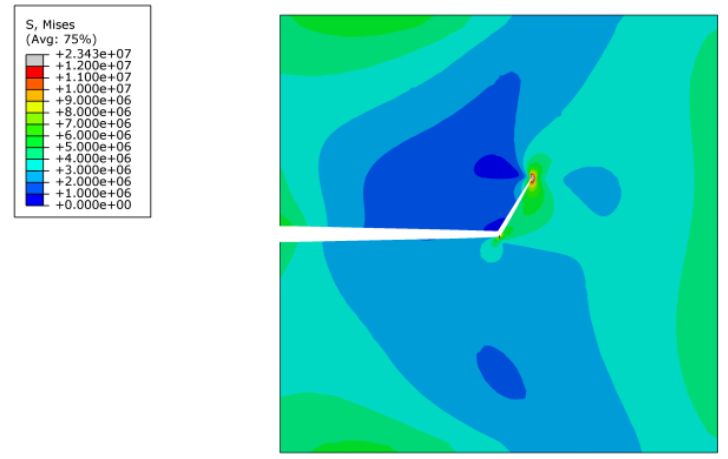

Fig. 9. the fracture propagation situation when the approach angle is $60^{\circ}$ and the tensile strength is $2 \mathrm{MPa}$.
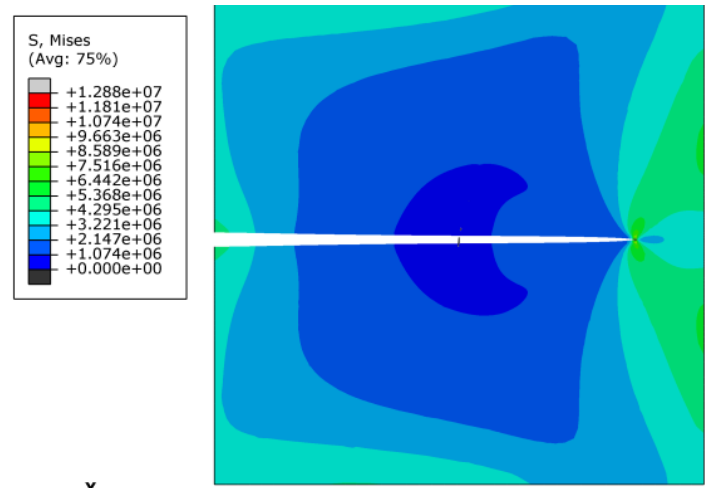

Fig. 10. the fracture propagation situation when the approach angle is $75^{\circ}$ and the tensile strength is $2 \mathrm{MPa}$.

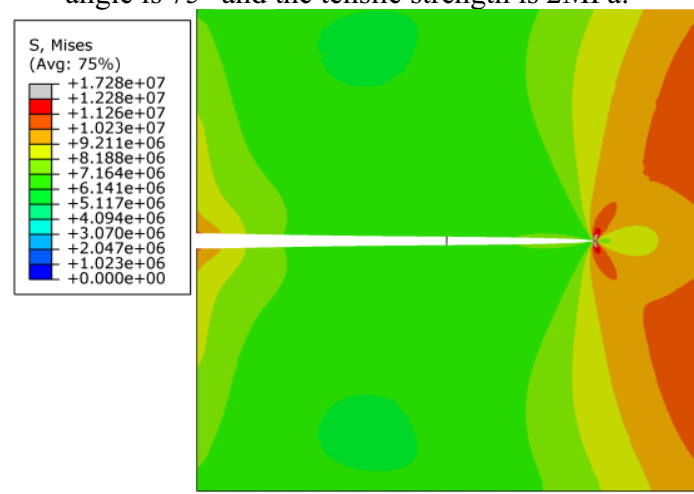

Fig. 11. the fracture propagation situation when the approach angle is $90^{\circ}$ and the tensile strength is $2 \mathrm{MPa}$.
Table 3. Propagation behavior of hydraulic cracks under different tensile strengths and different approach angles.

\begin{tabular}{|c|c|c|c|c|}
\hline $\begin{array}{r}\text { approach } \\
\text { angle } \\
\text { tensild } \\
\text { strength /MPa }\end{array}$ & 45 & 60 & 75 & 90 \\
\hline 1 & capture & capture & traverse & traverse \\
\hline 2 & capture & capture & traverse & traverse \\
\hline 3 & capture & traverse & traverse & traverse \\
\hline 4 & traverse & traverse & traverse & traverse \\
\hline
\end{tabular}

It can be seen from the above figure that the hydraulic fractures expand along the direction of the maximum horizontal principal stress when they do not encounter natural fractures. The tensile strength of the natural fractures is $2 \mathrm{MPa}$, and the approach angles are $45^{\circ}$ and $60^{\circ}$, the hydraulic fractures are captured by natural fractures, which continue to expand along the upper wing direction of the natural fractures; when the approach angle is $75^{\circ}$, the hydraulic fractures encounter natural fractures and then continue to expand through the natural fractures. The numerical simulation results in Table 3 show that when the approach angle is kept constant, as the tensile strength of natural fractures increases, the ability of hydraulic fractures to communicate with natural fractures becomes worse and worse, and it is easier to penetrate natural fractures and then expand along the direction of maximum horizontal principal stress. The possibility of connecting natural fractures to form a complex fracture network is reduced, mainly because as the tensile strength of natural fractures increases, the opening of natural fractures needs to overcome greater resistance, when the force generated by fracturing fluid is not enough to open natural fractures, The hydraulic fracture will penetrate the natural fracture and expand along the direction of the maximum horizontal principal stress; when the tensile strength of the natural fracture remains unchanged, as the approach angle increases, the ability of the hydraulic fracture to communicate with the natural fracture becomes worse and worse. It is easy to penetrate the natural fractures and expand along the direction of the maximum horizontal principal stress. The probability of connecting natural fractures to form a complex fracture network is reduced, mainly because as the approach angle increases, the opening pressure of the fracturing fluid on the natural fractures decreases, and the natural fractures are difficult to open.

\section{Conclusion}

(1) We simulate the influence of natural fractures in the rock on the propagation behavior of hydraulic fracturing fractures based on the cohensive unit of Abaqus finite element software.

(2) The results show that the three factors of approach angle, difference in ground stress, and tensile strength of natural fractures have important influence on the the 
propagation behavior of hydraulic fractures, any of the three factors increases, that will make it easier for hydraulic fractures to penetrate natural fractures and expand along the direction of the maximum horizontal principal stress, and reduce the probability of connecting natural fractures to form a complex network of fractures.

\section{References}

1. Chen Mian.Journal of China University of Petroleum(Edition of Natural Science), China.Reorientation and propagation of hydraulic fractures in shale gas reservoir.5,6( 2013).

2. Wei yuanlong, Yang chunhe,Guo yintong,et al. Chinese Journal of Rock Mechanics and Engineering,Experimental study on hydraulic fracture geometry of tight sandstone from Xujiahe group . 1,31(2016).

3. Heng shuai,Yang chunhe,Zeng yijin,et al. Chinese Journal of Geotechnical Engineering, Experimental study on hydraulic fracture geometry of shale. 7,8(2014).

4. Hou zhenkun, Cheng hanlie,Hai jinlong,et al. Journal of Yangtze River Scientific Research Institute, Fracture Mechanics Model of the Initiation and Growth of HydraulicFissures During Hydraulic Fracturing of Shale.5,3(2020).

5. Zhang jian, Wang jinyi,Jing tieya,et al. Journal of Changzhou University(Natural Science Edition Numerical Analysis of the Influence of Natural Fracture Angle on Hydraulic Fracturing [J]. 3,6(2020). 